\title{
A FÍSTULA ESÔFAGO-GÁSTRICA CERVICAL
}

\author{
CERVICAL ESOPHAGUS-GASTRIC FISTULA
}

\author{
Fausto Orsi, TCBC-RJ ${ }^{1}$ \\ Mônica Orsi, TCBC-RJ ${ }^{2}$ \\ Gustavo Carvalho, TCBC-RJ ${ }^{3}$ \\ Roberto Gonçalves, RCBC-RJ ${ }^{4}$ \\ Heitor Santos, RCBC- RJ ${ }^{5}$
}

\begin{abstract}
RESUMO: Objetivo: Analisar a presença de fístula esôfago-gástrica cervical nos pacientes submetidos a esofagectomias por câncer após reconstrução do trânsito digestivo com o estômago nas três vias: pré-esternal, retro-esternal e mediastino posterior. Método: Em um total de 180 pacientes portadores de carcinoma de células escamosas de esôfago torácico, tratados no Hospital Geral de Nova Iguaçu e no Hospital EMCOR, de agosto de 1968 a março de 2000, foram realizadas 97 esofagectomias e 70 (72,16\%) reconstruções do trânsito digestivo. $\mathrm{O}$ tratamento considerado foi essencialmente cirúrgico através da esofagectomia transpleural direita e da esofagectomia transhiatal. A anastomose esôfago-gástrica cervical foi realizada inicialmente em parede anterior do estômago e depois em parede posterior. Paralelamente, foram realizados estudos experimentais em cadáveres frescos no IML (Instituto Médico Legal) de Nova Iguaçu, para avaliação das dimensões das paredes gástricas e pesquisa de suas vascularizações. Resultados: A incidência de fístulas ficou reduzida a 7,69\%, quando se passou usar a parede posterior do estômago. A reconstrução do trânsito digestivo foi realizada em $52,86 \%$ pela via pré-esternal, $10 \%$ pela via retro-esternal e $37,14 \%$ pelo leito esofágico. As fístulas ocorreram em $20 \%$ dos pacientes ( 14 casos). Na via pré-esternal ocorreram $24,43 \%$ (9 casos), na via retro-esternal $42,85 \%$ ( 3 casos), e mediastino posterior 7,69\% ( 2 casos). ( $\mathrm{X} 2=3,39 ; \mathrm{p}=0,18$ ) A mortalidade operatória foi de 15,71\%, sendo a insuficiência respiratória sua maior causa.((X2=3,29; $\mathrm{p}=$ $0,19)$. A sobrevida em cinco anos foi de $13,5 \%$. Conclusões: A esofagectomia com anastomose esôfagogástrica cervical é o nosso método de escolha. Os melhores resultados foram obtidos com a execução da anastomose esôfago-gástrica cervical na parede posterior do estômago, e através do mediastino posterior.
\end{abstract}

Descritores: Câncer do esôfago; Fístula

\section{INTRODUÇÃO}

Este trabalho tem por precípuo escopo analisar a presença de fístula esôfago-gástrica cervical após esofagectomia parcial por carcinoma de células escamosas do esôfago torácico. Na recons- trução do trânsito digestivo, após esofagectomia quer por via transpleural direita ou transdiafragmática foi utilizado um tubo gástrico através de três vias: préesternal, retro-esternal ou pelo leito esofágico (mediastino posterior). Chamamos a atenção para a redução do número de fístulas esôfago-gástricas

1. Clínica Cirúrgica Fausto Orsi LTDA e Chefe do Departamento de Cirurgia Geral do Hospital EMCOR

2. Médica do Hospital da Piedade, RJ

3. Médico do Instituto Nacional do Câncer - INCA

4. Médico do Hospital Geral de Nova Iguaçu

5. Médico do Hospital Universitário São José

Recebido em 12/10/2001

Aceito para publicação em 22/08/2002

Trabalho realizado no Hospital Geral de Nova Iguaçu e no Hospital EMCOR 
cervicais quando utilizamos a parede posterior do estômago.

\section{MÉTODO}

Dos 180 pacientes portadores de carcinoma de células escamosas do esôfago torácico tratados de agosto de 1968 a março de 2000 no Hospital Geral de Nova Iguaçu e no Hospital EMCOR, 97 foram submetidos a esofagectomias. A reconstrução do trânsito digestivo foi feita em 70 casos $(72,16$ \%) (Tabela-1).

Não foram analisados os pacientes que possuíam câncer de esôfago cervical e adenocarcinoma de esôfago terminal e cárdia, já que merecem outro tipo de abordagem.

O tubo gástrico, que é confeccionado através da secção do estômago, da pequena curvatura ao fundo, é levado ao esôfago cervical por um túnel préesternal, retro-esternal ou ainda pelo leito esofágico. A anastomose é executada no fundo gástrico, na parede posterior, em dois planos de sutura de pontos separados e com fio de absorção tardia. Sutura-se a mucosa do esôfago à do estômago em um primeiro plano, e a serosa do estômago à adventícia-muscular do esôfago em um segundo plano ${ }^{1-3}$. Damos preferência pela técnica manual e não mecânica, não só pelas dificuldades técnicas e complicações, como também pelo elevado custo destas últimas. A seguir, o estômago próximo à anastomose a envolve parcialmente, e ainda é fixado a estruturas vizinhas, a fim de evitar tensões sobre a mesma ${ }^{4,5}$.

A vascularização do tubo gástrico agora fazse pela artéria gástrica direita e gastroepiplóica direita.

Com o objetivo de estudar a influência das dimensões e da vascularização das paredes anterior e posterior do estômago como elementos responsáveis pelas deiscências de anastomose esôfago-gástrica cervical, realizamos no Instituto Médico Legal (IML) de Nova Iguaçu, um estudo das medidas do comprimento da parede anterior e posterior do estômago em 10 cadáveres frescos. O tempo entre a entrada do corpo no IML e as mensurações das paredes gástricas foram de aproximadamente de duas horas.

Uma vez retirado o estômago, o mesmo foi esvaziado, lavado e medido, obedecendo a seguinte sequiência: distensão e fixação da peça. A partir do piloro e com o estômago fechado, marcamos na grande curvatura um ponto a $7 \mathrm{~cm}$ do mesmo. A seguir, com uma régua métrica, medimos a parede anterior e posterior do estômago a partir do ponto mais alto do fundo gástrico até este ponto na grande curvatura. Posteriormente, todos os estômagos foram abertos pela grande curvatura, distendidos e fixados à uma mesa. Passamos então, a medir todos os estômagos do ponto mais alto do fundo gástrico, pelas suas faces internas até o piloro.

\section{RESULTADOS}

Os resultados observados na Tabela- 2 comprovam que a parede posterior do estômago decorrente de sua maior distensibilidade, tem dimensões diferentes da parede anterior.

A Tabela-3 mostra a ocorrência de 17 deiscências de anastomose, das quais três fecharam e 14 evoluíram para fístulas. As três deiscências, por serem pequenas $(0,5 \mathrm{~cm})$, fecharam com simples uso de bastão de nitrato de prata, ou com uso da alimentação enteral por sonda, todos na via préesternal.

A Tabela-4 mostra a queda acentuada do número de fístulas quando usada a via de reconstrução do trânsito digestivo pelo mediastino posterior.

As fístulas cervicais maiores, em torno de 2 $\mathrm{cm}$, podem ser tratadas pela simples aproximação de suas bordas reavivadas, através de pontos separados ou através da técnica descrita a seguir.

A pele é cortada em uma extensão de $5 \mathrm{~cm}$ de ambos os lados da fístula e as bordas revertidas para o centro da mesma, onde são suturadas. Formase um tubo de pele com suas bordas superiores, inferiores e centrais suturadas, ficando a pele virada para dentro e o tecido celular subcutâneo exposto. A área cruenta agora é recoberta por enxerto de pele autógena, retirada da coxa com dermátomo.

Tabela 1 - Condutas Cirúrgicas (Esofagectomias $=97$ casos; Reconstrução do Trânsito Digestivo $=70$ casos).

\begin{tabular}{lrr}
\hline Esofagectomia sem toracotomia & 26 casos & $26,81 \%$ \\
Reconstrução com estômago & 26 casos & \\
Via mediastino posterior & 26 casos & $26,81 \%$ \\
Esofagectomia com toracotomia & 71 casos & $73,19 \%$ \\
Reconstrução com estômago & 44 casos & \\
Via pré-esternal & 37 casos & $52,86 \%$ \\
Via retro-esternal & 7 casos & $10,00 \%$ \\
\hline
\end{tabular}


Tabela 2 - Dimensões das paredes anterior e posterior do estômago.

\begin{tabular}{|c|c|c|c|}
\hline $\begin{array}{l}\text { Idade } \\
\text { (anos) }\end{array}$ & Parede & $\begin{array}{l}\text { Fechado } \\
\text { (cm) }\end{array}$ & $\begin{array}{l}\text { Aberto } \\
\text { (cm) }\end{array}$ \\
\hline \multirow[t]{2}{*}{9} & Anterior & 16 & 19 \\
\hline & Posterior & 18,5 & 21 \\
\hline \multirow[t]{2}{*}{28} & Anterior & 25 & 33 \\
\hline & Posterior & 26 & 34 \\
\hline \multirow[t]{2}{*}{43} & Anterior & 30 & 33 \\
\hline & Posterior & 31,5 & 36 \\
\hline \multirow[t]{2}{*}{39} & Anterior & 25 & 32 \\
\hline & Posterior & 27 & 34 \\
\hline \multirow[t]{2}{*}{66} & Anterior & 27 & 33 \\
\hline & Posterior & 30 & 35 \\
\hline \multirow[t]{2}{*}{38} & Anterior & 26 & 32 \\
\hline & Posterior & 27,5 & 33 \\
\hline \multirow[t]{2}{*}{30} & Anterior & 28 & 31 \\
\hline & Posterior & 29 & 33 \\
\hline \multirow[t]{2}{*}{28} & Anterior & 27 & 32 \\
\hline & Posterior & 29 & 34 \\
\hline \multirow[t]{2}{*}{35} & Anterior & 26 & 31 \\
\hline & Posterior & 28 & 33 \\
\hline \multirow[t]{2}{*}{30} & Anterior & 27 & 32 \\
\hline & Posterior & 29 & 34 \\
\hline
\end{tabular}

A morbidade e mortalidade pós esofagectomia, nesta série é semelhante a encontrada na literatura ${ }^{6}$.

A tabela 5 mostra que a insuficiência respiratória foi a principal causa de morte pósoperatória.

Apesar da predominância dos estádios III ( 80 casos - 44,48\%) e IV ( 85 casos - 47,23\%) a sobrevida em 59 pacientes acompanhados foi de $13,5 \%$ em cinco anos.

Tabela 3 - Complicações imediatas.

\begin{tabular}{lrr}
\hline Derrame pleural & 6 casos & $6,18 \%$ \\
Empiema pleural & 2 casos & $2,06 \%$ \\
Infecção de parede torácica & 5 casos & $5,15 \%$ \\
Insuficiência respiratória grave & 6 casos & $6,18 \%$ \\
Infarto do miocárdio & 3 casos & $3,09 \%$ \\
Mediastinites & 2 casos & $2,06 \%$ \\
Deiscências de anastomoses & 17 casos & $24,28 \%$ \\
a)- pré-esternal (37 casos) & 12 casos & $32,43 \%$ \\
b)- retro-esternal (7 casos) & 3 casos & $42,85 \%$ \\
c)- mediastino posterior (26 casos) & 2 casos & $7,69 \%$ \\
\hline
\end{tabular}

Tabela 4 - Complicações tardias.

\begin{tabular}{lrc}
\hline Estenoses & 3 casos & $4,28 \%$ \\
Fístulas esôfago-gástricas cervicais & 14 casos & $20 \%$ \\
a)- técnica pré-esternal & 9 casos & $24,43 \%$ \\
b)- técnica retro-esternal & 3 casos & $42,85 \%$ \\
c)- técnica mediastino posterior & 2 casos & $7,69 \%$ \\
\hline
\end{tabular}

$(X 2=3,39 ; p=0,18)$

\section{DISCUSSÃO}

Na história destes pacientes disfágicos, o que chamou nossa atenção, foi a presença constante de passado de alcoolismo, tabagismo e cuidados higiênicos orais precários. Tanto o tabaco como o álcool são considerados agentes carcinógenos, que provocariam mutações em genes supressores como o P53, presente em 50\% dos tumores malignos do esôfago ${ }^{7}$.

Em nossa série, as ressecções de carcinoma de células escamosas em qualquer porção do esôfago foram realizadas em maior número por esofagectomias via transpleural, pois seguíamos a seqüência traçada pela técnica de Nakayama ${ }^{8-10}$. Só posteriormente, viemos a empregar a via transdiafragmática.

$\mathrm{Na}$ reconstrução do trânsito digestivo utilizando o estômago, é importante salientar a confecção do tubo gástrico ${ }^{11}$ que tem início pela pequena curvatura, na altura do $5^{\circ}$ ramo da artéria gástrica esquerda, como preconiza Akyiama?

O tubo gástrico colocado pelo mediastino posterior nos trouxe um índice de complicações bem inferior.

Tabela 5 - Mortalidade pós-operatória.

\begin{tabular}{lrr}
\hline Via pré-esternal (37 casos) & 6 óbitos & $16,21 \%$ \\
Via retro-esternal (7 casos) & 3 óbitos & $42,85 \%$ \\
Via mediastino posterior (26 casos) & 2 óbitos & $7,69 \%$ \\
Total (70 casos) & 11 óbitos & $15,71 \%$ \\
Causa Mortis & & \\
Insuficiência Respiratória Grave & 6 casos & $54,54 \%$ \\
Infarto do Miocardio & 3 casos & $27,27 \%$ \\
Mediastinites & 2 casos & $18,18 \%$ \\
\hline
\end{tabular}

$(X 2=3,29 ; p=0,19)$ 
Estudos de angio-arquitetura arterial em estômagos humanos, realizada por Guerra e Rodrigues $^{12,13}$, identificaram quatro ou cinco áreas que dependem diretamente das artérias do mesmo nome, e que se comunicam entre si. Verificaram também a presença inconstante $(56,7 \%)$ de pacientes que apresentam ramos gástricos posteriores da artéria frênica inferior esquerda em posição retro-peritoneal, irrigando a parede posterior do estômago. Way et al. ${ }^{14}$ Michels $^{15}$ e Sabiston ${ }^{16}$, reconhecem que $60 \%$ das pessoas possui uma artéria gástrica posterior, originado-se do terço médio da artéria esplênica, e termina em ramos sobre a superfície posterior do corpo e fundo gástrico.

Observamos em nossa série, que a grande maioria das deiscências anastomóticas ocorrem na anstomose esôfago-gástrica anterior, e quase nunca na anastomose posterior. O ponto de fragilidade desta anastomose está em seus ângulos, que não suportam um aumento de pressão gástrica intratorácica durante um processo de tosse ou de um esforço respiratório.

Como foi observado em nosso estudo, a parede posterior do estômago apresenta dimensões diferentes da parede anterior, e com o conhecimento arterial acima descrito, passamos a fazer todas as anastomoses na parede posterior do estômago, com resultados muito superiores até então obtidos.

As anastomoses esôfago-gástricas cervicais sempre foram uma preocupação para os cirurgiões, devido a alta incidência de deiscências nas mesmas. Fatores de ordem anatômica do órgão e de ordem geral contribuem para a sua formação $0^{3,17-23}$.
Com a intensão de minimizar este problema, Hopkins et al. ${ }^{24}$, Wong et al..$^{25}$, Aquino et al. ${ }^{18}$, Sallum et $a l .{ }^{26}$ e Orringer et al. ${ }^{27}$ preconizaram a sutura mecânica para as mesmas. Em uma análise criteriosa dos trabalhos destes autores, ficou demonstrado que a incidência de fístula na anastomose esôfago-gástrica cervical não apresenta diferenças significativas entre suturas mecânicas e manual. Observou-se também, que além da dificuldade em se utilizar estes aparelhos devido a exigüidade do campo cirúrgico, o índice de estenoses com esta técnica foi bem maior ${ }^{5,28-32}$.

Assim, baseado em que não há diferença significativa no aparecimento de deiscência entre o uso da técnica manual e mecânica; na dificuldade de se trabalhar em campo cirúrgico limitado; no prolongamento do ato cirúrgico; no custo maior dos grampeadores, achamos que a sutura mecânica ao nível do esôfago cervical não oferece vantagens em relação a sutura manual por nós praticada.

Em uma análise estatística em relação às Tabelas 4 e 5 e calculadas as diferenças pelo teste X2 na Tabela-4, mostrou-se que, apesar das diferenças percentuais entre as diferentes técnicas cirúrgicas em relação às complicações tardias $(\mathrm{X} 2=3,39 ; \mathrm{p}=0,18)$ e em relação à mortalidade (Tabela-5), $(\mathrm{X} 2=3,29$; $\mathrm{p}=0,19)$, ambas não mostraram diferenças significativas.

Concluímos que os melhores resultados obtidos nas confecções destas anastomoses foram com aquelas realizadas na parede posterior do estômago, sendo a via preferencial do autor na reconstrução do trânsito digestivo, o mediastino posteior.

\begin{abstract}
Background: From August 1968 to March 2000 we attended 180 patients at Hospital Geral de Nova Iguaçu and at Hospital EMCOR, that had squamous cell esophagus cancer. The cervical esophagus gastric fistula was studied. Method: The treatment was essentially surgical, through transhiatal and transpleural esophagectomy. In the reconstruction of the digestive transit three approaches were used: pre-esternal, retro-esternal and posterior mediastinum, with their positives aspects and complications. Results: The number of leaks was reduced to 7,69\% when the posterior gastric wall was used. The postoperative mortality was $15,71 \%$ and respiratory insufficiency was the main cause. The five-year survival rate was 13,5\%. Conclusions: Cervical esophagus-gastric anastomosis was the procedure used in all cases, firstly in the anterior wall and finally in the posterior wall. The best results were obtained with cervical esophagus-gastric anastomosis in the posterior wall.
\end{abstract}

Key words: Esophagus cancer; Esophagus-gastric fistula 


\section{REFERÊNCIAS}

1. Kunzle JE, Ziliotto AJ - Estudos das anastomoses esofágicas cervicais após esofagectomias. Revista CBC - março/abril; 1985, XII, no 2 -58.

2. Levy W - Veruche über die resektion der speiseröhre. Arch Klin Chir, 1898, 56:839.

3. Chen J, Wei G, Shao L - A comparative study of cervical and thoracic anastomosis after esophagectomy for esophageal carcinoma. Chung Hua Chung Liu Tea Chih, 1996, 18:131-133.

4. Orsi FL, Pinotti HW - Condutas no tratamento cirúrgico do câncer de esôfago e da cárdia. Revista RBC, 1979 março / abril; - vol 69 (3/4):65-78.

5. Petrin G, Ruol A, Santi S. et al. - Anastomotic stenosis correlet with the use of mechanical staplers in the surgery of esophageal cancer. Ann Ital Chir, 1998, 693:513-519.

6. Swisher SG, Deford L, Merriman KW, et al. - Effect of operative volume on morbity, mortality and hospital use after esophagectomy for cancer. J. Thorac Cardiovasc Surg, 2000; 119: 1126-1134.

7. Akiyama H - Esophageal anastomosis. Arch Surg, 1979, 107:512-514.

8. Ellis FH.Jr - Treatment of carcinoma of the esophagus and cardia. Mayo Clin Proc, 1989, 64:945.

9. Nakayama K - Experiences os about 3.000 cases with cancer of the esophagus and cardia. Atlas of gastrointestinal surgery, 1966, 1:130.

10. Nakayama K - Upper and midthoracic esophageal cancer. Atlas of gastrointestinal surgery, 1968, 1:168.

11. Young MM, Dechamps C, Schleck CD, et al.: Esophageal reconstruction for benign disease: Early morbidity, mortality and functional results. Ann Thorac Surg, 2000, 70: 1651-1655.

12. Guerra AJ, Rodrigues H - Anatomia cirúrgica das artérias gástricas, seus territórios de predominância de distribuição e suas anastomoses. Revista RBC, 1982, março/abril de IX, ํㅡㄴ 2 - 50 .

13. Pierie JP, Graaf PW, Van Vroonhoven TJ, et al. - The vascularizacion of gastric tube as a substitute for the esophagus is affected by diameter. Dis esophagus, 1998, 11:231-235.

14. Way LW, et al.- Cirurgia - Diagnóstico e tratamento. 9a edição - 24: 334 - Guanabara Koogan, 1993.

15. Michels NA - Blood supply of the stomach and esophagus. Orgais Philadelphia JB Lippincott Company. 248; 1955.

16. Sabiston DC JR - Suplemento sangüíneo do estômago - arteriografia. Tratado de cirurgia. Guanabara Koogan - 1;716, 1993.

17. Ferreira EAB, Margarido NF, Saad Jr, et al.- Complicação em cirurgia. Robe. São Paulo. 1993; 25-3.

18. Aquino J L B, Ishida P, Muraro C P M, et al.Avaliação da sutura mecânica no esôfago cervical. Congresso do CBC -1993, Rio de Janeiro, 20; 47.
19. Urschel J D - Esophagogastrostomy anastomotic leaks complicating esophagectomy: a review. Am J Surg, 1995, 169:634-640.

20. Gurski R R, Schimer C C, Toneto J E, et al.- Delayed cervical anastomosis of the esophagus for esophageal carcinoma. Int Surg, 1997; 82:56-59.

21. Bai S, Chen 1, He M - A new technique of esophagogastrostomy above the aortic arch covering and suspending of the anastomotic area with mediastinal pleura flap. Chung Liu Tsa Chih, 1995,17:450-453.

22. Jacobi C A, Zieren J, Zieren HU, et al. - Is tissue oxigen tension during esophagectomy a predictor of esophagogastric anastomotic healing? J Surg Res, 1998, 74:161-164.

23. Choi HK, Law S, Chu KM, et al. - The value of neck drain in esophageal surgery: a randomized trial. Dis esophagus, 1998, 40-42.

24. Hopkins RA, Alexander JC, Postlethwait RW - Stapled esophagogastric anastomosis. Am J Surg, 1984, 147: 237-238.

25. Wong J, Cheund H, Lui R, et al.- Esophagogastric anastomosis perfomed with a stapler the occurrence of leakage and stricture. Surgery, 1987, 101:408-415.

26. Sallum RRA, Yamamuro EM, Cecconello, et al.- Sutura manual x mecânica na anastomose esofagogástrica cervical. Congresso Brasileiro do Colégio Brasileiro de Cirurgia Digestiva. 1996, Goiânia, 7:242.

27. Orringer M B, Marshall B, Iannettoni MD - Eliminating the cervical esophagogastric anastomotic leak with a side-to-side stapled anastomosis. J Thorac Cardiovasc Surg, 2000, 119: 277-288.

28. Bruns CJ, Gawenda M, Wolfgarten B, et al.- Cervical anastomotic stenosis after gastric tube reconstruction in esophageal carcinoma. Evaluation of patient sample 1989-1995. Langebecks Arch Chir, 1997, 382:154-158.

29. Trentino P, Pompeo E, Nofroni J, et al.- Predicative value of early post-operative esophagoscopy for ocurrence of benign stenosis after cervical esophagogastrostomy endoscopy, 1997, 29:840-844.

30. Miyashita M, Onda M, Okawa K, et al.- Endoscopic dexametasone injection following balloon dilatation of anastomotic stricture after esophagogastrostomy. Am J Surg, 1997, 174:442-444.

31. Collard J M, Romagnoli R, Goncette I, et al.Terminalized semimechanical side to side suture techinique for cervical esophagogastrostomy. Ann Thorac Surg, 1998, 65:814-817.

32. Van Oosterom FJ, Oosting J, Obertop H - A free peritoneal patch does not affect the leakage rate but increases stricture formation of a cervical esophagogastrostomy. Dig Surg, 1999,16: 379-384.

Endereço para correspondência:

Fausto Luiz Orsi

Rua Cel. Bernardino Melo, 1399 sala 501

26250-020 - Nova Iguaçu - RJ 